\title{
Humedad y temperatura en nidos naturales y artificiales de tortuga golfina Lepidochelys olivacea (Eschssholtz 1829)
}

\author{
Moisture and temperature in hatching of olive turtle Lepidochelys olivacea (Eschssholtz 1829) \\ Juan F. Arzola-González ${ }^{1}$ \\ ${ }^{1}$ Gestión Ambiental de Ecosistemas Costeros. Facultad de Ciencias del Mar, Universidad Autónoma de Sinaloa \\ Apartado postal 610, Mazatlán, Sinaloa; México \\ elarzola@hotmail.com
}

\begin{abstract}
The variation in moisture and temperature was analized in the hatching of olive ridley turtle Lepidochelys olivacea eggs, during the 1992-1993 season in "El Verde" beach (Sinaloa, Mexico). This variation was compared for different incubation environments (natural nests, corrals and polystyrene boxes). The results show that the average temperature was $33.6^{\circ} \mathrm{C}$ in natural nests, $32.8^{\circ} \mathrm{C}$ in corral and $32.1^{\circ} \mathrm{C}$ in boxes. Moisture percentage fell between $59.6 \%$ to $23.4 \%$ as calculated by the weight method and for saturation of $22.9 \mathrm{~mL} \mathrm{~g}^{-1}$ to $8.8 \mathrm{~mL} \mathrm{~g}^{-1}$. Hatching percentage was $67.9 \%$
\end{abstract}

in natural nests, $64.1 \%$ in corral and $46.9 \%$ in boxes. A granulometric study of sand showed differences in sand grain for nests areas. For this season the incubation average temperature was $32.7^{\circ} \mathrm{C}$; for this reason, the hatchlings were females. The moisture levels registered in each incubation environment in this experiment apparently were not a determinant factor for the hatching success.

Key words: Moisture, temperature, incubation, nests, turtle

\section{Introducción}

En la actualidad las tortugas marinas se encuentran clasificadas como especies amenazadas o en peligro de extinción, según acuerdos de la Convención para el Comercio Internacional de Especies de Fauna y Flora Silvestre. Sin embargo, paralelamente a las medidas legales adoptadas por las instituciones responsables de su manejo, desde hace más de treinta años se han establecido campamentos de conservación y protección en las principales playas de anidación de tortugas cuya función principal es la protección de los huevos, vigilando los nidos naturales o trasladándolos a corrales o a cajas de incubación (Márquez 1978, Ríos \& Briseño 1985).

Durante el desarrollo de los embriones influyen algunos factores ambientales como humedad, temperatura, salinidad y tamaño del grano de la arena; los cuales, entre otros, determinan el éxito en la incubación (Arzola \& Armenta 1994). En particular, la humedad y la temperatura, aún siendo variables independientes y sin presuponer sobre una la acción de la otra, ambas están íntimamente relacionadas. Además, el efecto combinado de estos factores se inicia desde que los huevos son depositados en el nido hasta la emergencia de las crías (Zamora 1990).
El contenido de agua en el medio tiene influencia en el intercambio de este líquido entre el sustrato y el huevo (Bautista 1992). Un nivel excesivo disminuye el intercambio de agua, y en caso de inundación se llega a perder todo el nido. Por el contrario, la poca humedad causa resequedad y ésta tiene efectos negativos en la supervivencia, ya que los huevos se deshidratan, desecan y colapsan (McGehee 1979, Galicia et al. 1988 ${ }^{1}$, López 2002). Al igual que la humedad, la temperatura es considerada un factor de gran importancia en el proceso de incubación. Existe en la bibliografía, una amplia serie de informes que reportan la influencia fundamental de esta variable en la determinación del sexo (Naranjo 1988², Arzola \& Armenta 1994, Garduño \& Cervantes 1996), debido a su relación directa con la diferenciación sexual de los embriones (Mrosovsky \& Pieau 1991). Por lo anterior, el presente estudio tiene como propósito analizar las

\footnotetext{
${ }^{1}$ Galicia P, E Hernández, C López \& R Nieves. 1988. Influencia de la humedad de incubación sobre el porcentaje de avivamiento en tortuga golfina y laud en el Playón de Mexiquillo, Michoacán, durante la temporada de anidación 1987-1988. V Encuentro Interuniversitario sobre las Tortugas Marinas en México.

${ }^{2}$ Naranjo G. 1988. Características del ambiente de incubación natural y su influencia en el avivamiento de los nidos de tortuga negra (Chelonia agassizi) en las playas de Colola y Maruata. V Encuentro Interuniversitario sobre las Tortugas Marinas en México.
} 
posibles consecuencias de la humedad y la temperatura en nidos naturales y artificiales de la tortuga golfina Lepidochelys olivacea (Eschssholtz 1829).

\section{Material y métodos}

La playa de anidación El Verde se localiza en la costa sur de Sinaloa, México $\left(23^{\circ} 23^{\prime}-23^{\circ} 26^{\prime} \mathrm{N}\right.$ y $106^{\circ} 31^{\prime}$ $106^{\circ} 34^{\prime} \mathrm{W}$ (GPS GP 38 Garmin); presenta una extensión de aproximadamente $18 \mathrm{~km}$ de anidación. Al norte limita con promontorios rocosos extensos con escasas líneas de playa y al sur se interrumpe hasta el inicio del desarrollo turístico del puerto de Mazatlán.

Después de la ovoposición de la hembra, se realizó inmediatamente el traslado de los huevos al corral (nidos a una profundidad de $45 \mathrm{~cm}$ en zona de playa aledaña al campamento) y a las cajas de poliuretano, de acuerdo con los criterios de Márquez et al. (1990). En total se mantuvieron 30 nidos en observación, de los cuales diez se depositaron en cajas de poliuretano dentro de una sala de incubación en la playa El Verde; diez fueron transferidos al corral y los diez restantes se dejaron in situ, con el fin de comparar los resultados de las dos técnicas de protección.

La humedad se determinó con frecuencia semanal, siguiendo el método de McGehee (1979); se tomaron muestras de $100 \mathrm{~g}$ de cada sitio de nido, se pesaron en una balanza digital, se secaron en un horno durante 24 horas a $100^{\circ} \mathrm{C}$ y después se volvieron a pesar para determinar la cantidad de humedad perdida. La humedad se expresó como porcentaje de humedad en peso (diferencia, en peso entre muestra húmeda y seca sobre el peso de muestra seca por 100). La lectura de la temperatura fue cada 24 horas y se instaló un termosensor procurando ubicarse en el centro de la nidada. Los sensores se conectaron a un teletermómetro Sensortek modelo BAT 12 con una precisión de $0,1^{\circ} \mathrm{C}$. Adicionalmente, se hizo un estudio granulométrico en los nidos naturales y de corral para realizar la determinación del tamaño del grano de arena según Fowler (1979), el cual consiste en multiplicar la sumatoria del porcentaje en peso presente en cada intervalo de tamaño (frecuencia) multiplicado por el punto medio de cada intervalo y dividido entre el peso total de la muestra. El éxito de eclosión es considerado como el número de crías que eclosionaron y emergieron a la superficie del nido y se estimó sustrayendo el número de crías (vivas y muertas) y el número de cascarones vacíos dentro del nido $\mathrm{y}$ dividiendo estas diferencias del total del tamaño de la nidada. La determinación de las fases embrionarias se realizó de acuerdo a los criterios establecidos por Márquez (1978) en las siguientes fases: Huevo blanco, huevo rosa, fase I, II, III, IV y crías muertas. A los datos de humedad, temperatura y del grano de arena se les aplicó las pruebas estadísticas de normalidad (Lillieford), homocedasticidad (Bartlett), ANOVA de una vía y para la granulometría, la prueba de t-Student (Zar 1996) Además se realizaron correlaciones entre las variables de humedad y la eclosión y de temperatura con eclosión por tratamiento.

\section{Resultados y discusión}

Los datos obtenidos de la humedad fueron expresados en porcentaje en peso (Tabla 1), resultando en nidos naturales con valores de 56,6 a 37,2\%, en corral de 53,6 a $31,5 \%$, mientras que en cajas fue de 44 a 23,4\%. La Fig. 1 muestra la pérdida de humedad paulatina, pero en nidos naturales y de corral se registró un incremento aproximadamente a los 20 días de incubados en 8,8\% (naturales) y 11,8\% (corral), debido a precipitaciones locales. Los datos de humedad resultaron a través de una ANOVA de una vía, encontrándose alguna diferencia significativa entre los tratamientos (ANOVA de una vía, $P<0,0001$ ) en el contenido de humedad.

Sin embargo, la humedad presente en el sustrato incubatorio no puede ser considerado como un factor único de influencia en el porcentaje de eclosión; esta variable puede entonces ser alterada por otros factores físicos y tener efectos distintos en el desarrollo

Tabla 1

Variación de la humedad por peso (\%) en nidos de Lepidochelys olivacea en la playa EI Verde, Sinaloa

Variation in moisture by weight (\%) in nests of Lepidochelys olivacea in El Verde beach, Sinaloa

\begin{tabular}{lccc}
\hline & \multicolumn{3}{c}{ Humedad (\%) } \\
\cline { 2 - 4 } & Naturales & Corral & Cajas \\
\hline Máxima & 59,6 & 53,6 & 44,0 \\
Mínima & 37,2 & 31,5 & 23,4 \\
Promedio & 46,6 & 40,0 & 31,3 \\
Desv. est. & 8,55 & 7,6 & 8,7 \\
Varianza & 73,1 & 56,0 & 75,9 \\
\hline
\end{tabular}




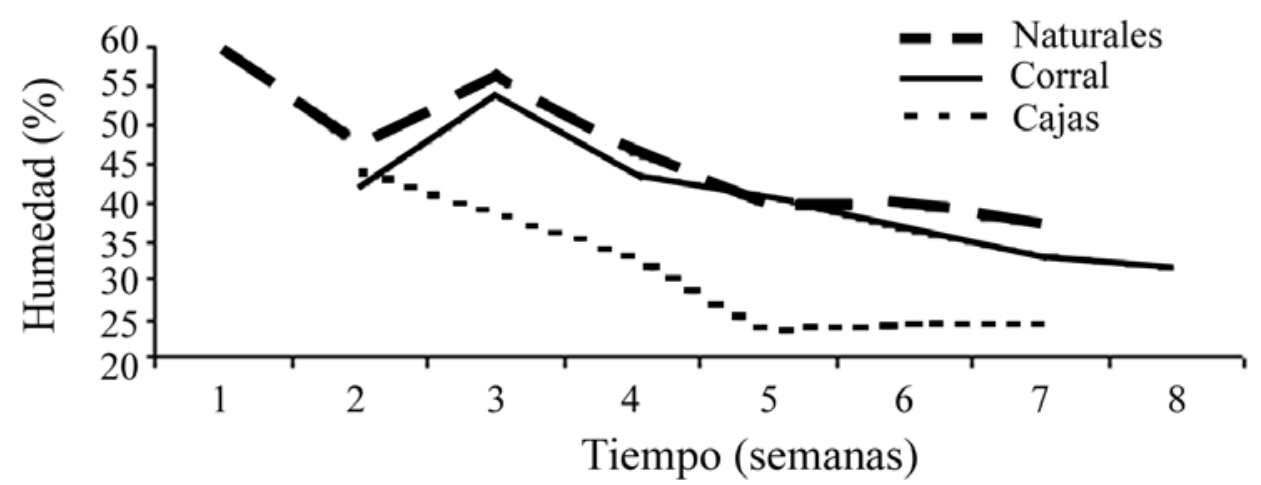

Figura 1

\section{Porcentaje de humedad por peso (\%) en nidos de Lepidochelys olivacea en la playa El Verde, Sinaloa}

Moisture percentage by weight (\%) in nests of Lepidochelys olivacea in the El Verde beach, Sinaloa

embrionario de estos organismos; McGehee (1979) sugiere que los efectos de humedad en la arena varían con el tamaño del huevo, siendo posible que exista una inclinación a la pérdida de humedad con el medio circundante en huevos de menor tamaño que los grandes; Packard et al. (1987) indican que a 0\% de humedad los embriones se deshidratan, siendo así, que la humedad de la arena puede ser considerada como critica durante la incubación; López (2002) señala que para contrarrestar la falta de humedad en los nidos, estos deben localizarse de 10 a 30 m retirados de la línea de marea.

Se encontró una temperatura ambiente promedio de $30^{\circ} \mathrm{C}$, mientras que entre los tratamientos asignados resultó en $32,7^{\circ} \mathrm{C}$. La Tabla 2 muestra los valores de máxima, mínima y promedio de la temperatura en los nidos de corral, naturales y en cajas. En nidos naturales, sin embargo, se mantuvo en una media de $34,4^{\circ} \mathrm{C}$, con una máxima de $36,9^{\circ} \mathrm{C}$ y una mínima de $31,9^{\circ} \mathrm{C}$, alcanzando los valores más altos de temperatura entre los tratamientos, aunque se registró una interrupción en la variable como consecuencia de una marejada extraordinaria (Fig. 2). En corral, el promedio se registró en $32,9^{\circ} \mathrm{C}$, con una variación máxima y mínima de $34,9^{\circ} \mathrm{C}$ a $28,2^{\circ} \mathrm{C}$, respectivamente; por último, en cajas la media fue $32,6^{\circ} \mathrm{C}$ con un intervalo de $34,8^{\circ} \mathrm{C}$ a $30,5^{\circ} \mathrm{C}$. Además, sobre la línea de playa donde se ubicaron los nidos naturales y de corral, se realizaron ciclos de 24 horas (cada 2 h) para determinar la variación térmica diurna, registrando una temperatura máxima de $35^{\circ} \mathrm{C}$ y una mínima de $32,5^{\circ} \mathrm{C}$.
Tabla 2

Variación de la temperatura en nidos de Lepidochelys olivacea en la playa El Verde, Sinaloa

Variation of temperature in nests of Lepidochelys olivacea in El Verde beach, Sinaloa

\begin{tabular}{lccc}
\hline & \multicolumn{3}{c}{ Temperatura $\left({ }^{\circ} \mathrm{C}\right)$} \\
\cline { 2 - 4 } & Naturales & Corral & Cajas \\
\hline Máxima & 36,9 & 34,9 & 34,8 \\
Mínima & 31,9 & 28,2 & 30,5 \\
Promedio & 34,4 & 32,9 & 32,6 \\
Desv. est. & 1,35 & 1,11 & 1,19 \\
Varianza & 1,84 & 1,23 & 1,42 \\
\hline
\end{tabular}

El principal factor en la determinación del sexo de Lepidochelys olivacea para esta área, sería la temperatura, mientras el contenido de humedad y el tamaño del grano de arena, sólo parecen provocar pequeñas variaciones, las cuales no fueron significativas, presentándose una tendencia natural a obtener sólo hembras, ya que el promedio de la temperatura fue de $33^{\circ} \mathrm{C}$ entre los nidos. Al respecto, algunos autores han demostrado que temperaturas mayores o igual a $31,2^{\circ} \mathrm{C}$, afectan la determinación del sexo, produciendo 100\% hembras (Aguilar 1987, Parckard et al. 1987, Plumer \& Snell 1988). Los resultados obtenidos en las crías producidas, permiten llevar a cabo un mejor manejo de las tortugas marinas, entre ellos: estudiar la proporción natural de sexos en las diferentes playas, efectuando registros térmicos durante la incubación de los huevos en las principales playas de anidación sin el sacrificio de las 


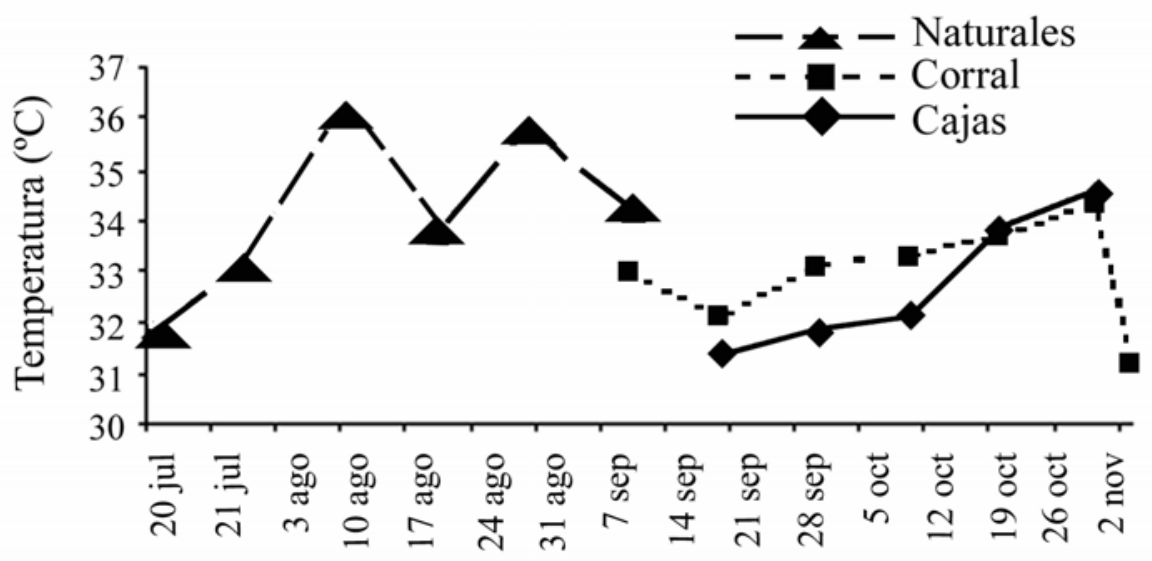

Tiempo (semanas)

Figura 2

Variación de la temperatura en nidos naturales, corral y en cajas de poliuretano de Lepidochelys olivacea en la playa El Verde, Sinaloa

Variation of the temperature in natural nests, corral and boxes of Lepidochelys olivacea in El Verde beach, Sinaloa

crías, además de planificar con ello un uso más racional de las tortugas. Inclusive, Van der Heiden et al. (1988) y Aguilar (1987) señalan que el período sensible para la determinación del sexo durante la incubación ocurre en los días del 14 al 21-22, a una temperatura umbral de 30 a $32^{\circ} \mathrm{C}$.

No se encontró una diferencia significativa de la temperatura entre los nidos naturales (ANOVA, $P=$ $0,924)$, mientras que en nidos transferidos al corral registraron una diferencia significativa (ANOVA, $P=$ 0,0456) y por último, la temperatura en las cajas resultaron significativamente diferentes (KruskalWallis, $P=0,002$ ).

En el análisis granulométrico, la playa El Verde presentó un tamaño de la arena de $0,5 \mathrm{~mm}$ a $0,062 \mathrm{~mm}$ de diámetro, destacando una predominancia de 0,25 $\mathrm{mm}$, clasificándose como arena de grano medio (escala Wentworth). En la Fig. 3 se muestra que la franja de playa con mayor densidad de nidos se ubicó en arena con grano grueso (59\%) tendiendo a grano medio. En los nidos de corral se encontró una mayor proporción de granos finos (53\%). Estos resultados coincidieron con lo reportado por Ríos \& Briseño (1985) para esta área, al determinar la presencia de arena de grano grueso con predominancia a grano medio con valores

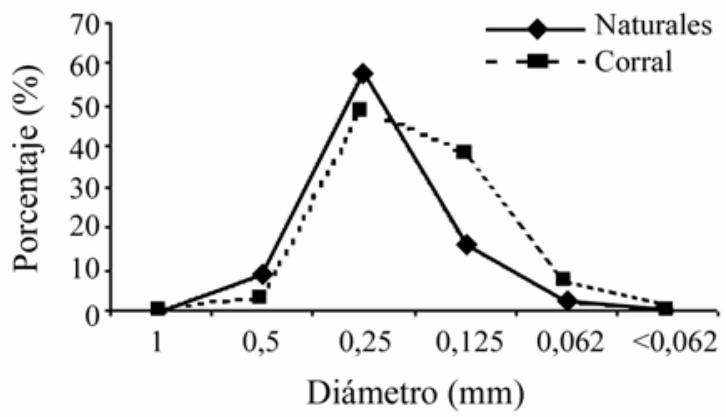

Figura 3

Análisis granulométrico (en mm) para la playa El Verde, Sinaloa, donde comúnmente desova Lepidochelys olivacea

Granulometric analysis (in mm) for El Verde beach, Sinaloa, where often breeds Lepidochelys olivacea

de 0,5 a 0,125 mm. No se encontró una significancia en el tamaño del grano de arena en la zona de los nidos naturales y de corral (t-Student, $P=0,873$ ). Sin embargo, la relación que existe en el tamaño del grano de arena permite una mejor distribución del oxígeno entre los espacios intersticiales y los embriones, aunque los resultados indicaron un mínimo porcentaje de tamaños por debajo de $0,062 \mathrm{~mm}$ (0,1\%); estos correspondieron a arcilla y limos, los cuales podrían 
provocar compactaciones muy importantes en la arena pudiendo reducir la difusión del oxígeno, el drenaje o la emergencia de las crías en los nidos. Sin embargo, este mecanismo natural no influyó de manera determinante en el porcentaje de eclosión en los tratamientos, ya que la diferencia en el éxito de avivamiento fue de 3,8\% entre nidos naturales y de corral.

Se incubó un total de 2966 huevos, divididos en tres tratamientos: 1071 en nidos naturales, 926 en corral y 969 en cajas de poliuretano, obteniéndose un porcentaje de eclosión general del 59,8\%, es decir, 1775 crías vivas y por tratamiento, en 67,9\%, 64,1\%, y 46,9\% respectivamente (Fig. 4). Es posible que el mayor porcentaje de eclosión en los nidos naturales sea provocado por la diferencia significativa en la influencia de la temperatura y el contenido de la humedad, no así en nidos de corral y en cajas. Además, la manipulación de los huevos durante la incubación puede contribuir en el éxito de la eclosión, ya que una vez polarizado el disco germinal en el embrión, cualquier ligero cambio o inclinación en él afectará su desarrollo posterior, principalmente en nidos sembrados en las cajas, ya que se obtuvo menos del $50 \%$ de la eclosión. Además, es probable que el esfuerzo que se realiza para cuidar y proteger a los nidos naturales y de corral, sea mayor ya que se requiere vigilar la playa constantemente; sin embargo, sería factible que los resultados obtenidos fueran mejores desde el punto de vista biológico, en comparación con las cajas de poliuretano.

Al analizar los datos de las variables de temperatura y humedad con respecto a la eclosión, se encontró que los valores promedio de la temperatura fueron muy similares en los nidos naturales, de corral y en cajas, registrando entre los tratamiento una diferencia de tan solo $1,5^{\circ} \mathrm{C}$, no influyendo quizás directamente en el éxito de la eclosión. Sin embargo, el comportamiento de la humedad fue muy similar en los nidos naturales y de corral, pero no así en cajas, donde la pérdida de humedad fue mayor y continua durante el desarrollo embrionario, provocando quizás el menor porcentaje de eclosión entre los nidos (46,9\%). Además, el análisis granulométrico indicó que la composición del grano de arena no fue diferente entre los nidos naturales y de corral, así como tampoco en sus porcentajes de eclosión. Por su parte, los coeficientes de correlación $\left(r^{2}\right)$ entre el porcentaje de eclosión y la temperatura resultaron muy bajos en los tratamientos: 0,12\% (naturales), 0,05\% (corral) y 0,002\% (cajas); al igual

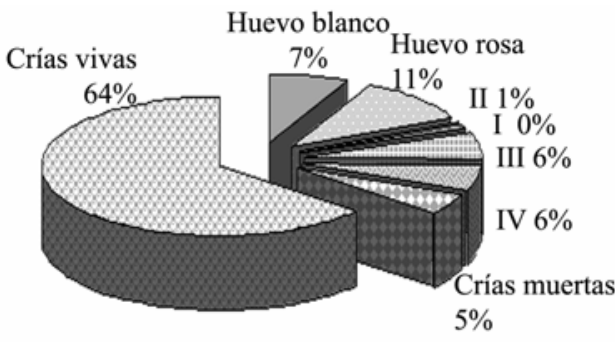

a)
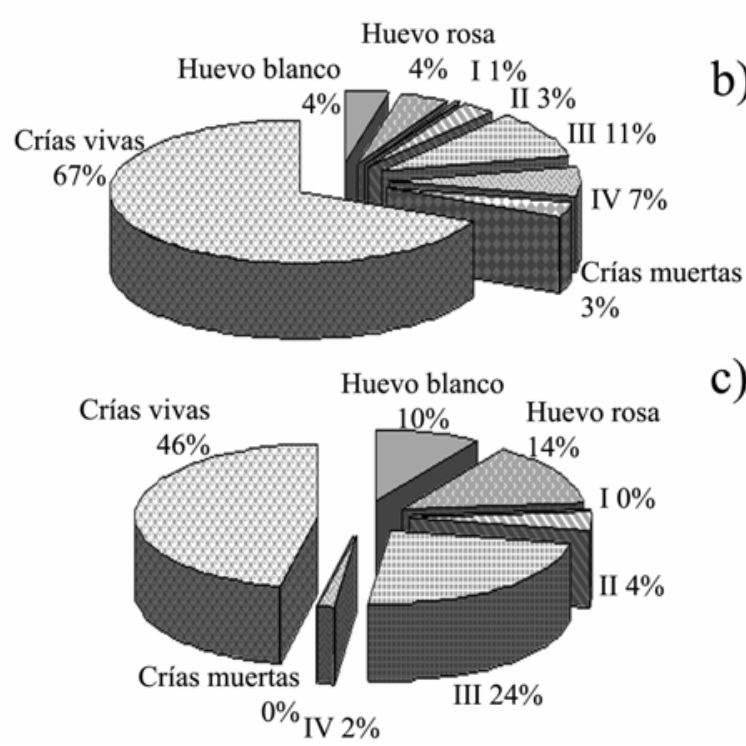

c)

Figura 4

Análisis de embriones interrumpidos durante la incubación y crías vivas (\%), en nidos naturales (a), corral (b) y en cajas (c) de Lepidochelys olivacea para la playa El Verde, Sinaloa

Analysis of broken embryos during incubation and life breeding on natural nests (a), corral (b) and boxes (c) of

Lepidochelys olivacea in El Verde beach, Sinaloa

que la supervivencia con la humedad en peso $\left(\mathrm{r}^{2}=0,05\right.$, 0,49 y 0,09, respectivamente), no se descarta la importante influencia que ejercen dichas variables en el éxito de eclosión de huevos de tortugas marinas como lo han indicado algunos autores (Torrey $1978 \mathrm{y}$ McGehee 1979).

La etapa III fue la más sensible en nidos naturales y de corral. Una hipótesis probable es considerar que el cambio de temperatura haya influido durante la última quincena de incubación, ya que se registró en el ambiente una baja de temperatura, pudiendo ocasionar un decremento brusco en la cámara de incubación, 
provocando así un posible colapso en muchos de los embriones en desarrollo. Además, los huevos ubicados en las capas inferiores del nido, tienen una menor disponibilidad de oxígeno y por consiguiente el riesgo de perecer por asfixia es mayor. Aunado a lo anterior, estos embriones se encuentran soportando la presión de los huevos de las capas superiores.

Por otra parte, en los embriones que se encontraron en la última etapa de desarrollo (IV) y que corresponde a un tiempo de incubación de 37 a 40 días aproximadamente, o bien en crías que terminaron su desarrollo, pero que no alcanzaron a emerger del nido, quizás esta mortalidad natural sea provocada por los aportes excesivos de líquido amniótico al momento de la eclosión masiva y de la compactación de las cáscaras y huevos que impidieron la salida de las crías débiles o pequeñas del nido.

$\mathrm{Al}$ analizar los embriones interrumpidos en las cajas, se registró un mayor porcentaje para las primeras etapas (H.B., H.R., I y III). Sin embargo, en la cámara de incubación se presentó un decremento en la temperatura ambiental en el último período de la embriogénesis, teniendo como resultado un descenso en la temperatura de $1^{\circ} \mathrm{C}$, y quizás esto, provocó la muerte de una cantidad considerable de embriones. No se encontró ninguna cría muerta y se obtuvo una diferencia de 3,8\% en etapa final, comparado con nidos dejados en la playa.

El tiempo de incubación estimado hasta el momento de la primera emergencia de las crías correspondió a un promedio de 45 días con una duración máxima de 47 diás y una mínima de 42 días; además, el horario de emergencia de las crías se registró entre las 23:00 p.m. y las 5:00 a.m. Como resultado de la temperatura registrada durante el desarrollo embrionario, se presentó una disminución de tres días en la incubación. Esto coincide con otros autores que también han determinado que un incremento en temperatura, origina una disminución en el tiempo de incubación (Márquez et al. 1976, Mrosovsky \& Yntema 1980, Aguilar 1987).

\section{Literatura citada}

Aguilar H. 1987. Influencia de la temperatura de incubación sobre la determinación del sexo y duración del período de incubación en la tortuga lora (Lepidochelys kempi). Tesis de Biología, Instituto Politécnico Nacional, México, 58 pp.
Arzola F \& J Armenta. 1994. Análisis comparativo de dos factores ambientales en nidos naturales $y$ nidos trasladados y su efecto en crías de tortuga golfina, Lepidochelys olivacea en la playa El Verde, Mazatlán, Sinaloa, durante la temporada 1992-1993. Tesis de Biología, Instituto Tecnológico de Los Mochis, México, 72 pp.

Bautista HF. 1992. Relación de la humedad del ambiente incubatorio en el avivamiento de Lepidochelys olivacea (tortuga golfina) en las playas de La Escobilla, Oaxaca. Tesis de Biología. Universidad Nacional Autónoma de México, México, 63 pp.

Flowler L. 1979. Hatching success and nest predation in the green sea turtle, Chelonia mydas, at tortuguero, Costa Rica. Ecology 60: 946-955.

Garduño M \& E Cervantes. 1996. Influencia de la temperatura y la humedad en la sobrevivencia en nidos in situ y en corral de tortuga carey (Eretmochelys imbricata) en Las Coloradas, Yucatán, México. INP. SEMARNAP. Ciencia Pesquera 12: 90-97.

López M. 2002. Densidad y características de anidación de la tortuga golfina (Lepidochelys olivacea), en Cabo Pulmo, B.C.S., (2000). Tesis de Biología Marina. Universidad Autónoma de Baja California Sur, México, 64 pp.

Márquez R, A Villanueva \& C Peñaflores. 1976. Sinopsis de datos biológicos sobre la tortuga golfina Lepidochelys olivacea, 66 pp. Instituto Nacional Politécnico. Subsecretaría de Pesca, México.

Márquez R. 1978. Instructivo para la protección de las tortugas marinas. S.I.C. Instituto Nacional de la Pesca. Serie de Divulgación 2:1-33.

Márquez R, J Vasconcelos, J Sánchez, S Sánchez, J Díaz, C Peña, D Ríos \& A Villanueva. 1990. Manual de operación de campamentos tortugueros. 115 pp. Instituto Nacional de la Pesca, México.

McGehee M. 1979. Effects of moisture on eggs and hatchlings of loggerhead sea turtles (Caretta caretta). Department of Biological Sciences, University of Central Florida. Herpetology 3: 251-258.

Mrosovsky N \& L Yntema. 1980. Temperature dependence of sexual differentiation in sea turtles: Implications for conservation practices. Conservation Biology 18: 271280.

Mrosovsky N \& M Pieau. 1991. Transitional range of temperature, pivotal temperature and thermosensitive stage for sex determination in reptiles. Amphibia-Reptilia 12: 169-179. 
Packard G, M Packard, K Miller \& T Boardman. 1987. Influence of moisture, temperature and substrate on snapping turtle eggs and embryos. Ecology 68: 983-993.

Plumer M \& H Snell. 1988. Nest site selection and water relations of eggs in the snake, Opheodrys aestirus. Copeia 1988(1): 58-61.

Ríos D \& R Briseño. 1985. Informe final de actividades, campamento tortuguero "El Verde", Sinaloa. 22 pp. Instituto Nacional de la Pesca, Centro Regional de Investigación Pesquera (CRIP). Mazatlán, Sinaloa, México.

Torrey, M. 1978. Morfogénesis de los vertebrados, 776 pp. Editoral Limusa, México.
Van der Heiden A, R Briseño \& D Ríos. 1988. A simplified method for determining sex hatchling sea turtles. Copeia 1988(3): 779-782.

Zamora R. 1990. Relación de la humedad del ambiente incubatorio en el avivamiento de Chelonia agassazi en la playa de Cólola, Michoacán. Tesis de Biología. Universidad de San Nicolás de Hidalgo. Michoacán, México, 94 pp.

Zar J. 1996. Biostatistical analysis. 662 pp. Prentice-Hall, Englewood Cliffs.

Recibido el 27 de junio de 2006 y aceptado el 22 de junio de 2007 